\title{
APPLIED INFOCOMMUNICATION SYSTEMS AND THEIR MODELS
}

\author{
Alexander Sotnikov \\ Saint-Petersburg State University of Telecommunications prof. M.A. Bonch Bruyevich, \\ St. Petersburg, Russia, \\ adsotnikov@mail.ru \\ Gallia Katasonova \\ Saint-Petersburg State University of Telecommunications prof. M.A. Bonch Bruyevich, \\ St. Petersburg, Russia, \\ 1366galia@mail.ru
}

\begin{abstract}
Methods for formal description of informational interaction in complex info-communicational systems and its models are investigated. Main notions and terms are defined. Complex of multilevel models for applied info-communicational systems for telemedicine are proposed
\end{abstract}

Keywords - Information interaction, info-communication, systems, telemedicine, models

\section{INTRODUCTION}

The development of modern telecommunications is characterized by several key tendencies, including the following:

- Telecommunication and information technologies have penetrated deep into applied spheres, becoming a key functional element of applied branch systems. The telecommunication technologies spread to the spheres of government and administration, trade and distribution, producing "trademark" terms like "e-Government", “ecommerce", etc.

- The merging of the information (meaningful content) and telecommunications has produced a new entity: an infocommunicational system (ICS), which changes the principles of ICS analysis, development and construction, and the setup and provision of infocommunicational services (ICSe). Tele-education and telemedicine (TM) are the most typical realizations of the concept of "infocommunication service".

Telemedicine presents an interdisciplinary synthesis of healthcare, telecommunications and information technologies (IT), which are traditionally used for specific methods of analyzing and designing autonomous systems and services, creating and providing services based on internal organizational and functional principles and technologies. TM systems (TMS) are a combination of several heterogeneous interdependent systems, thus falling under the definition of "complex systems" (CS) [1]. Effective methods of extending general systemic demands to CSs to specific subsystems, the optimization of inter-systemic interaction, remain to be developed. Problems become especially relevant for socially oriented ICS (education, healthcare), with severe cost limitations.

The main problem is the lack of descriptive and analytical methods in the applied sphere, which would be applicable both to IT and telecommunications industry, which means that the criteria for TMS design cannot be correctly formulated. The aim of the project is studying the principles of information interaction within the ICS; classification of ICS according to a number of criteria; creation of complex ICS models aimed at quantitative estimation of parameters within the applied area of healthcare.

\section{THEORETICAL BASICS OF INFOCOMMUNICATIONS}

The key categories and concepts for infocommunications are those of "information", "information interaction", "information process", "information object". As a fundamental scientific term, information lacks a concise and universally accepted definition [2]. This lack of unity means that the category is insufficiently developed and can be defined and interpreted in new ways.

Information interaction (II). The category of interaction reflects the mutual impacts of various objects, their mutual causality and change of state. The information process, as a realization of a specific II, is defined by a number of authors as “... non-energetic interaction of objects, characterized by transfer (generation and internalization) of ideal categories (senses, meanings, images, emotions)" or "... interaction of objects leading to the change in the knowledge possessed by at least one of them" [2].

\section{A. Key concepts and definitions}

An information system deals with "information objects": manifestations $\{\langle A\rangle,\langle B\rangle, \ldots\}$ of entities 


\section{$\{A, B, \ldots\}[3]$.}

The information is transferred, when the signal transmitting an image (notion) from the varifold thesaurus of the source system $A$ into the varifold thesaurus of the target system $B$ is changed

$$
\langle A\rangle^{\xi_{A}} \stackrel{\text { Signal }}{\Rightarrow}\left\langle\langle A\rangle^{\xi_{A}}\right\rangle^{\xi_{B}}
$$

The information is received when a new image of the source is formed within the varifold thesaurus of the target system.

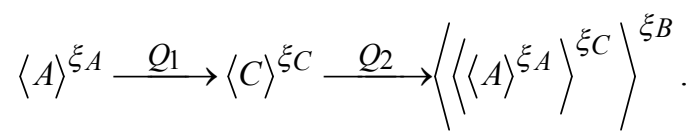

Information impact is the influence of the "source" A on the state of the "target" B, which manifests itself in the change of the image $\langle B\rangle$, seen in the variety of elements within the thesaurus of the "source" $\langle B\rangle\rangle_{A}$. Since the "source" $\xi_{A}$ and "target" $\xi_{B}$ thesauri are different, the original image in the internal thesaurus of $\langle B\rangle^{\xi_{B}}$ and the image of $\langle B\rangle^{\xi_{A}}$ in the "source" thesaurus which serves as an observes, are also different. This can lead to errors, or inaccurate representation of the object in the thesaurus of the "observer".

Information exchange is the receiving and transmission of signals leading to the mutual alteration of images $\langle A\rangle \xi_{B}$ and $\langle B\rangle \xi_{A}$ of the exchange participants. This can be caused by alteration (expansion) of the participants' thesauri $\xi_{A}$ and $\xi_{B}$.

Information interaction is the mutual change of images of own systems of $\langle A\rangle^{\xi_{A}}$ and $\langle B\rangle^{\xi_{B}}$, leading to the change of images $\langle A\rangle \xi_{B}$ and $\langle B\rangle \xi_{A}$ in the other participants.

Information system (IS) is a system containing "information" and providing it to the "user". A necessary condition is as follows: "The necessary components of an IS are: the user and the potential information". A sufficient condition is "The user and the potential information form an IS". IS are made up of elements which are information images $\langle A\rangle$ of the real (material and immaterial) entities $A$ and possess "information significance".

"Information significance" is the property of representing the entity, which requires a descriptive method containing a set of basic "meanings", immanent to the entity. "Information significance" should not be confused with "value" or "usefulness"; these properties are related to the users and the possibility of satisfying their need of information.

The formalized set of "meanings" is the $\xi_{A}$ thesaurus. The entity item has a number of discernible states, which are perceived by the observer as a set of images of an object, each having its own "meaning". The number of states determines the potential information carried by the object. When the observer acquires an image of the object (by means of perception and recognition), the potential information is actualized on the basis of the information representation of the object. The potential presence of information in system $\boldsymbol{A}$ is determined by the set of discernible states of the system and the varifold system thesaurus $\xi_{A}$.

Perception of the information transferred occurs when the receiver $R$ acquires a new image of system $\boldsymbol{A}$ in the varifold receiver thesaurus $\left\langle\langle A\rangle \xi_{A}\right\rangle \xi_{R}$.

User $\boldsymbol{U}$ is a person, object or process capable of perceiving images $\langle S\rangle \xi_{S}$ and possessing its own receiver thesaurus $\xi_{U}$.

Potential information is the set of discernible states of the entity $S$ in the set of representations $\langle S\rangle \xi_{S}$ based on the thesaurus $\xi_{S}$, possessing information significance.

Actual information is the representation (image) $\left\langle\langle S\rangle \xi_{S} \xi_{U}\right.$ in the thesaurus $\xi_{U}$ of the user $\boldsymbol{U}$.

Information System $\boldsymbol{S}$ :

$$
\left\{S, \xi_{S},\langle S\rangle^{\xi_{S}}, U, \xi_{U},\left\langle\langle S\rangle \xi_{S}\right\rangle^{\xi_{U}}\right\}
$$

is the combination comprised of: system entities, users, the system thesaurus and user thesauri, the set of information representations of the system (potential information), and the set of system images (representations in user thesauri).

Communication System C:

$$
\left\{C, \xi_{\mathrm{C}},\langle C\rangle^{\xi_{C}}, Q^{\xi_{A} \xi_{C}}, Q^{\xi_{C} \xi_{B}}\right\}
$$

is the combination of consecutive transformations

$$
\stackrel{Q_{12}^{\xi_{A} \xi_{C}}}{\longrightarrow}, \stackrel{Q_{23}^{\xi_{C}} \xi_{B}}{\longrightarrow},
$$

ensuring the representation of the set of source images $\langle A\rangle$ in the set of target images $\langle B\rangle$ via the set of states (images) $\left\langle C \xi_{C}\right.$ of signal $C$ given required precision.

Telecommunication System $\boldsymbol{T}$ :

$$
\left\{C, \xi_{C},\langle C\rangle \xi_{C,} Q^{\xi_{A} \xi_{C}}, Q^{\xi_{C} \xi_{B}}\right\}
$$

is the combination of consecutive transformations $Q^{\xi_{A} \xi_{C}}, Q^{\xi_{C} \xi_{B}} \quad$ signals and their representations, regardless of the spatial location of the source $A$ and target $B,(A \in D, B \in D, D \neq A \cap B)$.

Info-communication System $\boldsymbol{F}$ :

$$
\begin{gathered}
\left\{S, \xi_{S},\langle S\rangle \xi_{S}, U, \xi_{U},\left\langle\langle S\rangle \xi_{S} \xi_{U}, C, \xi_{C},\right.\right. \\
\left.\langle C\rangle^{\xi_{C}}, Q^{\xi_{A} \xi_{C}}, Q^{\xi_{C}} \xi_{B}\right\}
\end{gathered}
$$

is a combination including the entities of the information system $\boldsymbol{S}$ and telecommunication system $\boldsymbol{T}$.

\section{B. ICS Classification}

Within the framework provided by these definitions, 
it is possible to classify ICS according to a number of criteria which characterize different aspects of the interaction between the system, the user and the information, important from the point of view of the analysis and subsequent design of ICS $[4,5]$. In this context, the spatial distance between the users is of no consequence, and the notions of ICS and IS are the same thing.

"Information placement". Information objects (representations of entities) are an integral ICS element. The entities themselves (carriers of potential information) are not always elements of the ICS.

The presence or absence of entities within the ICS is the first classification criterion, which determines the way in which entities and/or information objects (i.e. potential information) are obtained and adequately presented, which has important repercussions.

1. Entities possessing potential information are "inside" the ICS, and their informational representations are placed there as well. The inclusion of an object into a system (Fig. 1) presupposes the coincidence between the subset of the object thesaurus with the set of the system thesaurus $\xi_{A} \in \xi_{S}$, resulting in the coincidence between representations $\langle A\rangle \xi_{A} \equiv\langle A\rangle \xi_{\text {S. }}$.

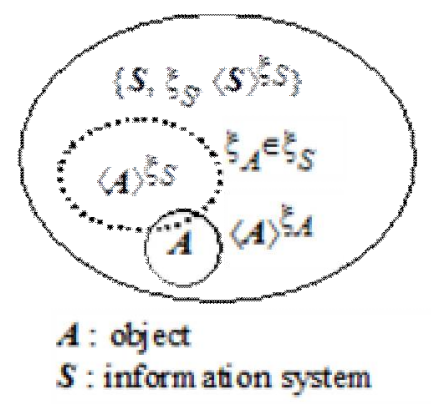

Fig.1. Information "inside" the system.

2. Entities are "outside" the system (Fig. 2). In this case, the system and object thesauri are linked by the correlation $\xi_{S} \in \xi_{A}$, and only an approximation of images $\langle A\rangle_{A} \approx\langle A\rangle \xi_{S}$ is possible. It is determined by the proximity of the thesauri $\xi_{S}, \xi_{A}$, i.e. the information is incorrectly represented by the system.

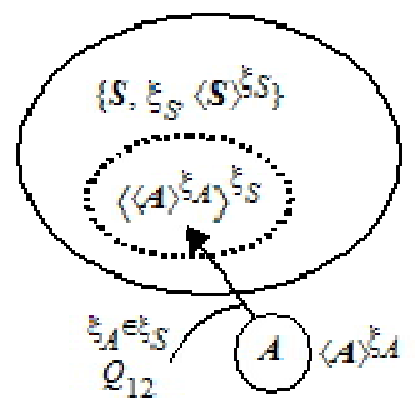

Fig.2. Information "outside" the system.

The relationship between the user and IS. This is the second criterion for ICS classification. The user can be either "inside the system" or "outside the system". The terms "inside" and "outside" reflect the interaction possibilities between the system and use thesauri, rather than spatial or topological characteristics.

1. The user is "within the system" (Fig. 3), when:

a) The system and user thesauri are entirely identical $\xi_{S} \equiv \xi_{U}$. This is a case of confluence, when the user is identified with the system and can no longer be regarded as the "receiver" of the information, because he possesses the entirety of images arising from the varifold system thesaurus, while the IS cannot serve as a source of information, the definition is violated and the usefulness of the IS is lost.

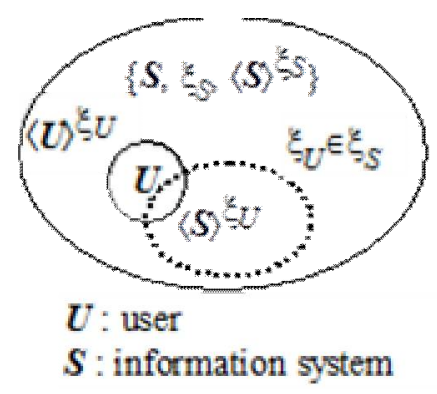

Fig.3. User inside the system.

b) The user thesaurus can be arbitrarily changed (expanded) by means of adding elements of the system thesaurus, which is initiated by the user, whose thesaurus can then be "reconciled" with the system thesaurus $\xi_{S} \approx \xi_{U}$ to a degree.

c) The system thesaurus can be arbitrarily changed by the user.

In cases when "the user is inside the system", there is no operation of mutual transformation of thesaurus elements $Q^{\xi_{S} \xi_{U}}$.

Instead, one subset is represented in the other $\boldsymbol{F}$ : $\boldsymbol{S} \rightarrow \boldsymbol{U}$, i.e. it is possible to perceive the image adequately. The degree of adequacy is determined by the degree of thesaurus coincidence, i.e. the degree of proximity between sets $\xi_{S}, \xi_{U}$.

2. The user is outside the system (Fig. 4), when:

a) The user $\xi_{U}$ and system $\xi_{S}$ thesauri are autonomous $\left(\xi_{S} \leftarrow x \rightarrow \xi_{U}\right)$ and cannot be reconciled to an arbitrary degree "at the user's initiative", but only "at the request of the system", as a result of providing information to the user. 


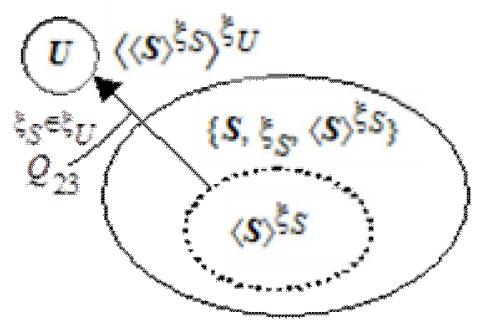

Fig.4. User outside the system.

When the user is "outside the system", the thesaurus transformation operation becomes significant

$\left(Q^{\xi_{U} \xi_{S^{\prime}}=\xi_{U} \cup \xi_{S^{\prime}}}\right)$, where $S^{\prime} \in S$, or more rarely $Q^{\xi_{S} \xi_{U}}$, which corresponds to the change (expansion) of the thesaurus, provides for the expansion of entity space and for the greater precision in the perception of the image of the object $\langle A\rangle \xi_{A}$ in the thesaurus of the user $\left\langle\langle A\rangle \xi_{A} \xi_{U}\right.$. The correlation between the user, object and system thesauri $\left(\xi_{U}, \xi_{A}, \xi_{S}\right)$ is crucial for the classification of IS, since the image is created in the varifold thesaurus, i.e. the image is derived from the thesaurus $A \Rightarrow \xi_{A} \Rightarrow\langle A\rangle^{\xi_{A}}$. The most complicated case is when the user and the entities are "outside" the system (Fig. 5). This produces the two-way exchange, which is described in the following way in the formal notation.

Let the entity, the $I S$ and the user with their thesauri and representations accordingly be:

$$
\left\{A, \xi_{A},\langle A\rangle \xi^{\xi_{A}}\right\},\left\{S, \xi_{S},\langle S\rangle \xi_{S}\right\},\left\{U, \xi_{U},\langle U\rangle^{\xi_{U}}\right\}
$$

then the image of the entity created in the user thesaurus corresponds to the two-way exchange between the entity, the IS and the user, defined by the sequence

$$
\begin{aligned}
& \langle A\rangle^{\xi^{A}} \stackrel{Q^{\xi^{A} \xi^{S}}}{\longrightarrow}\left\langle\langle A\rangle^{\xi^{A}}\right\rangle^{\xi^{S}} \stackrel{Q^{\xi^{S} \xi^{U}}}{\longrightarrow} \\
& \left.\mid\left\langle\langle A\rangle^{\xi^{A}}\right\rangle^{\xi^{S}} \stackrel{Q^{\xi^{S} \xi^{U}}}{\stackrel{\xi^{A}}{\longrightarrow}}\left\langle\langle A\rangle^{\xi^{U}}\right\rangle^{\xi^{U}}\right\rfloor,
\end{aligned}
$$

where the expression in brackets describes the "reproduction" of image $\langle A\rangle \xi_{A}$ in the image $\left\langle\langle A\rangle \xi_{A}\right\rangle_{U}$ created in the varifold thesaurus of the user.

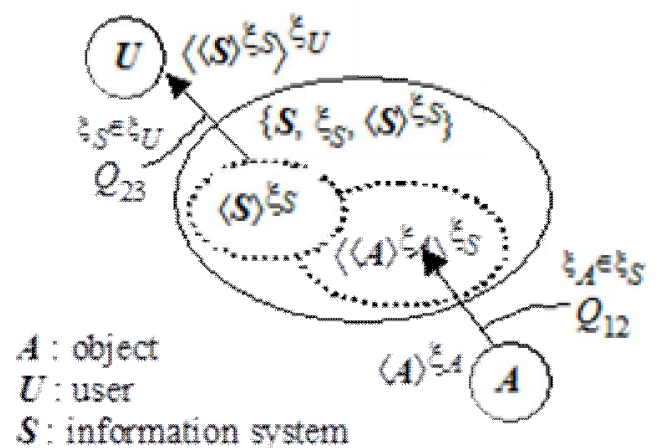

Fig.5. User and data are "outside" the ICS.

On the basis of the first two criteria, the most important characteristics of the structural and functional organization of the ICS can be classified; they manifest themselves in its type (Fig. 6). Type $A$ stands for one user, information "inside" the system, user "outside" the system. Type Al stands for multiple user, information "inside: the ICS, the users "outside" it. Type B stands for one or a limited number of users, information and user "inside" the ICS. Type B1 stands for multiple users, information and users "inside" the system. Type $C$ stands for two users, information possessed by the users, users "outside" the ICS. Type D stands for user "inside", information "outside" the system. Type E stands for two (multiple) users and information "inside" the ICS. Type $F$ is a combination of systems of types $\mathrm{A}-\mathrm{C}$.

Depending on the combination of constituent system types, there are several standard subtypes (F1 - F4).

\section{Applied ICS}

A special ICS class has no means of influencing the entities generating the representations, but originating in areas outside the "sphere of information": healthcare, education, defense, administration etc.

This class ICS should be regarded separately and defined as "applied ICS". It is characterized by the following properties:

1. Entities (real objects) are by definition "outside" the system.

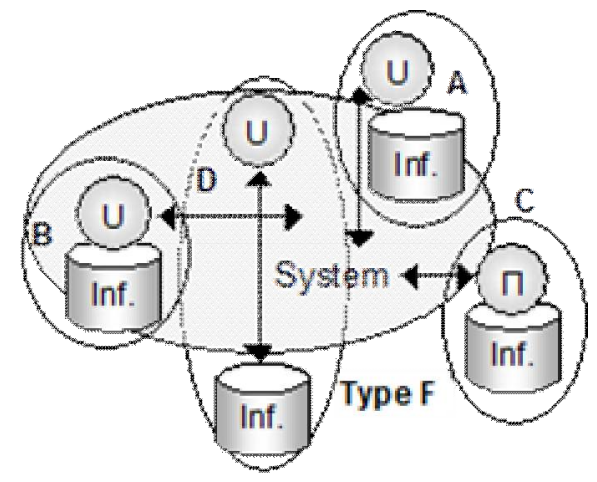


Fig.6. Types of IC systems

2. The system and entity thesauri are correlated as follows: $\xi_{S} \in \xi_{A}$.

3 . It is impossible to change the entity thesaurus by means of IS, i.e. $\left|Q^{\xi_{S} \xi_{A}}\right|$.

4. The information is inaccurately represented by the ICS, since only approximations of information representations are possible $\left.\langle A\rangle^{\xi_{A} \approx\langle A}\right\rangle^{\xi_{S}}$, caused by the proximity of thesaurus pairs $\xi_{S} \xi_{A}$ and $\xi_{S} \xi_{U}$.

An external attribute of "applied ICS" is the strict requirement for the information and user to be "outside the system" (Type C); which can also be non-strict (Types A, A1, C, D, F), when the user possesses the information. Applied ICS are usually not formalized to a great degree, and so present the most difficulties for system design, requiring new descriptive mechanisms.

\section{INFO-COMMUNICATION SYSTEM MODEL}

The first model describes the ICS as a whole, defining its limits.

The domain-based infocommunication model

The least generic and abstract model is the "domain model" (DM) (Fig. 7), which makes it possible to identify ICS components, define the inter-component interfaces and consider the information interaction processes.

There are three domains within the DM, associated with three relatively independent, albeit closely linked, types of activity. The Physical Domain (PD) is mostly concerned with energy processes and the interaction of material objects.

The situation analysis and intellectual activity producing evaluations and solutions is the product of mental and psychic activity of the Cognitive Domain (CD).

According to the DM, the sphere of telecommunications lies on the boundary between the physical and information domains. The perception of physical reality, represented by ID entities, is linked with information interaction and occurs on the border of ID and CD. Thus, infocommunications embrace all three domains, although at present, the cognitive domain remains insufficiently explored.

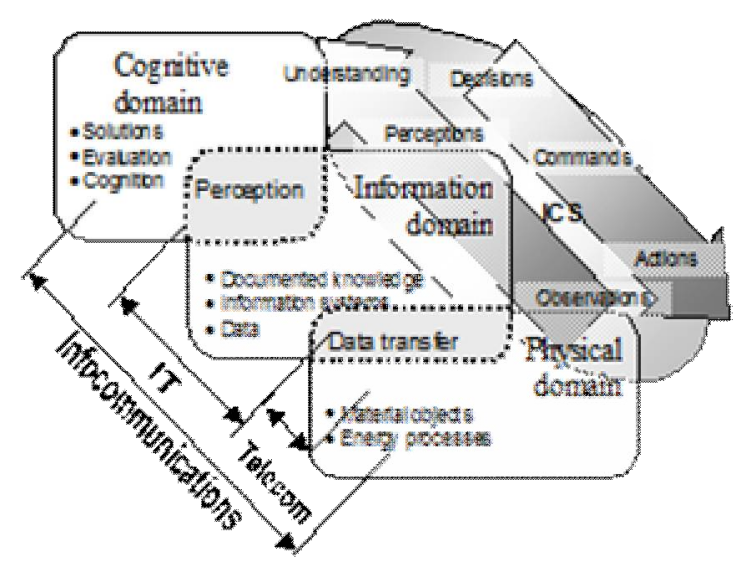

Fig.7 - The domain model of infocommunications.

The general view of elementary interaction between two information systems within the ID is a unidirectional transfer/receiving of the representation of the object of PD, described as follows:

$$
\left\langle\left\langle A_{n}\right\rangle^{\xi_{A n}}\right\rangle^{\xi_{C} m} \stackrel{Q_{22}^{\xi_{2}} C^{k}}{\longrightarrow}\left\langle\left\langle A_{n}\right\rangle^{\xi_{A n}}\right\rangle^{\xi_{C^{k}}}
$$

The information interaction occurs by means of exchanging "messages", which are the subsets of the set of information representations $\left\langle A_{1}\right\rangle^{\xi_{A n}}$ of the object $\boldsymbol{A}_{n}$, transferred between IS $C_{m}$ and $C_{K}$. Thus, the interaction of several IS within the ID is described as follows:

$$
\mid\left\langle\left\langle\left.\left\langle\left.\left\langle A_{n}\right\rangle^{\xi A_{n}}\right|_{n=1, . . N}\right\rangle^{\xi C^{m}} \underset{Q_{22}^{\xi C^{k} \xi C^{m}}}{\stackrel{Q^{m}}{Q_{22}^{\xi C^{m} \xi C^{k}}}}\right|^{k=1, . K}\right.\right.
$$

The information process is a combination of elementary information interactions within the ID. Expression (5) represents the "domain model", a description of information interaction linked to the nature of three domains.

Let $\left\{A_{1}, \ldots A_{6}\right\} \in \boldsymbol{A}$ : the set of objects in the PD, $\left\{C_{i}^{j}\right\} \in C$ : the set of objects in the ID, where $i=\{1, \ldots, 9\}, \quad j=\{1, \ldots, 5\}, \quad$ while the subsets of representations $\left\{C_{m}^{i}\right\}$ are within $m$ autonomous IS active within the ID.

Thus, for objects $A_{n}$ and consumers $B_{k}$ generally: 


$$
\begin{aligned}
& \left\langle A_{n}\right\rangle^{\xi A_{n}} \stackrel{Q_{12}^{\xi A n \xi C^{m}}}{\longrightarrow}\left\langle\left\langle A_{n}\right\rangle^{\xi A_{n}}\right\rangle^{\xi C_{n}^{m}} \\
& \stackrel{Q_{23}^{\xi C_{n}^{m} \xi B k}}{\longrightarrow}\left\langle\left\langle C_{n}^{m}\right\rangle^{\xi C_{n}^{m}}\right\rangle^{\xi B k} .
\end{aligned}
$$

Expression (6) represent the model of information interaction of two subjects of $\mathrm{CD}$, expressed in terms of ID system representations and PD objects. The representation of object $A_{1}$ in the user thesaurus $B_{I}$ serves as the basis for making decision $\left\langle R_{l}\right\rangle^{\xi_{B l}}$ by subject $\left(B_{l}\right)$ of $\mathrm{CD}$.

$$
\left\langle\left\langle\left\langle A_{1}\right\rangle^{\xi_{A_{1}}}\right\rangle^{\xi_{1} C_{1}^{1}}\right\rangle^{B_{1}} \stackrel{\Theta B_{1}}{\Rightarrow}\left\langle R_{1}\right\rangle^{\xi_{B_{1}}},
$$

where $\Rightarrow$ decision making operator.

The whole sequence of forming the image of object $A_{1}$ in the ID, its perception by subject $B_{1}$ in the CD, decision making and transfer via the ID to subject $B_{4}$ in the $\mathrm{CD}$, is described as follows:

$$
\begin{aligned}
& \left\langle\left.\left\langle\langle A 1\rangle^{\xi A_{1}}\right\rangle^{\xi C_{1}^{1}}\right|^{B_{1}} \stackrel{\Theta B_{1}}{\Rightarrow}\langle R 1\rangle^{\xi B 1} \stackrel{Q_{32}^{\xi B 1 \xi C_{7}^{4}}}{\Rightarrow}\left\langle\langle R 1\rangle^{\xi B_{1}}\right\rangle^{\xi C_{7}^{4}}\right. \\
& \left.\stackrel{Q_{23}^{\xi} C_{7}^{4} \xi B 4}{\langle}\left\langle C_{7}^{4}\right\rangle^{\xi C_{7}^{4}}\right\rangle^{B 4},
\end{aligned}
$$

This expression represents the information interaction model for CD subjects in terms of ID system representations, $\mathrm{PD}$ objects and $\mathrm{CD}$ subject decisions.

\section{CONCLUSION}

The main disadvantage of traditional approaches is the omission of the user and user-based applied processes from the ICS, which makes it difficult to study the information generation and utilization process in IS. A formal analysis of information interaction of ICS components, their basic properties and characteristics, makes it possible to identify different ICS types. The suggested classification criteria are based on the characteristic features of interaction between the ICS, the user and the information.

The approach described above makes it possible to make the transition from subjective evaluations to formulating the challenges and tasks of the quantitative analysis and synthesis of less formalized applied telemedical ICS.

\section{REFERENCES}

[1] Sotnikov A.D., Arzumanyan M.Y. "Competitive advantages of companies in information economy", St.
Petersburg State Polytechnical University Journal. Economics. №4 (102), 2010, pp.100-105.

[2] Sotnikov A.D. "Principles of the Applied Area Analysis in Healthcare Infocommunication Systems". In: Proceedings of Higher Educational Establishments in Communications, No.171, 2004, pp. 174-183.

[3] Sotnikov A.D., "Structural and Functional Organization of Telemedicine Services in Applied Infocommunication System", St Petersburg: 2007, pp. 1242.

[4] Sotnikov A.D., "Info-Communication Systems and their Respective Models for Healthcare Providers", Information and Control Systems. №3, 2008, pp. 46-53 